\title{
Sequential and counter-selectable cassettes for fission yeast
}

\author{
Hanna Amelina ${ }^{\dagger}$, Vera Moiseeva ${ }^{\dagger}$, Laura Catharine Collopy, Siân Rosanna Pearson, Christine Anne Armstrong
} and Kazunori Tomita* (D)

\begin{abstract}
Background: Fission yeast is one of the most commonly used model organisms for studying genetics. For selection of desirable genotypes, antibiotic resistance cassettes are widely integrated into the genome near genes of interest. In yeasts, this is achieved by PCR amplification of the cassette flanked by short homology sequences, which can be incorporated by homology directed repair. However, the currently available cassettes all share the same tef promoter and terminator sequences. It can therefore be challenging to perform multiple genetic modifications by PCR-based targeting, as existing resistance cassettes in strains can be favored for recombination due to shared homology between the cassettes.

Results: Here we have generated new selection cassettes that do not recombine with those traditionally used. We achieved this by swapping the tef promoter and terminator sequences in the established antibiotic resistance MX6 cassette series for alternative promoters and/or terminators. The newly created selection cassettes did not recombine with the tef-containing MX6 cassettes already present in the genome, allowing for sequential gene targeting using the PCR-based method. In addition, we have generated a series of plasmids to facilitate the C-terminal tagging of genes with desired epitopes. We also utilized the anti-selection gene HSV-TK, which results in cell death in strains grown on the drug 5-Fluoro-2'-deoxyuridine (FdU, Floxuridin or FUDR). By fusing an antibiotic resistance gene to HSV-TK, we were able to select on the relevant antibiotic as well as counter-select on FdU media to confirm the desired genomic modification had been made. We noted that the efficiency of the counter selection by FdU was enhanced by treatment with hydroxyurea. However, a number of DNA replication checkpoint and homologous recombination mutants, including rad $3 \Delta, c d s 1 \Delta$, rad54 $\triangle$ and rad55 , exhibited sensitivity to FdU even though those strains did not carry the HSV-TK gene. To remove counter-selectable markers, we introduced the Cre-loxP irreversible recombination method. Finally, utilizing the negative selectable markers, we showed efficient induction of point mutations in an endogenous gene by a two-step transformation method.
\end{abstract}

Conclusions: The plasmid constructs and techniques described here are invaluable tools for sequential gene targeting and will simplify construction of fission yeast strains required for study.

Keywords: Schizosaccharomyces pombe, DNA replication, Point mutation, Gene disruption and insertion, Thymidine kinase, FUdR, HA, Flag, PK tagging, Zeocin

\footnotetext{
* Correspondence: k.tomita@ucl.ac.uk

${ }^{\dagger}$ Equal contributors

Chromosome Maintenance Group, UCL Cancer Institute, University College

London, Paul O'Gorman Building, Huntley Street, London WC1E 6DD, UK
} 


\section{Background}

Fission yeast Schizosaccharomyces pombe is a popular model organism for fundamental biological research [1]. A haploid life cycle along with efficient homologous recombination system and simple reproductive processes make it an excellent system to study genetics. In yeast, genes can be deleted and replaced with selection markers readily, simply by inducing homology directed repair with a selection cassette amplified with primers that contain short, flanking sequences homologous to a target gene [2, 3]. A strain of induced genotype can be isolated simply by plating transformants onto corresponding selection plates. Generated strains can be further crossed to isolate multiple genetic alternations that can be selected for using combination of appropriate markers.

In fission yeast, commonly used drug selection cassettes are kanMX6, natMX6, hygMX6 (hphMX6) and bleMX6 $[2,4,5]$. These cassettes are composed of a promoter and terminator from the fungus Ashbya gossypii and genes conferring selectable resistance to G418 (geneticin or neomycin), nourseothricin (ClonNat or Nat), hygromycin B and bleomycin respectively. Auxotrophic gene cassettes, such as $\mathrm{ura}^{+}$from S. pombe and LEU2 from Saccharomyces cerevisiae have also been extensively used in fission yeast $[6,7]$. However, when using auxotrophic cassettes, strains must have mutations in the corresponding endogenous gene, which could affect cell metabolism, stress response and cell growth. Thus, use of antibiotic resistance markers has become standard for yeast organisms, as the working strain does not require any particular auxotrophic genetic background. However, as these antibiotic cassettes all share the same promoter and terminator sequences, it is difficult to perform sequential gene targeting due to the shared homology [5]. This issue is usually solved by genetic cross between two mutant strains. However, this strategy is not feasible for mutants dysfunctional in mating, meiosis or germination. In some cases the mating is successful, but it is difficult to obtain desired genotype in the offspring if genetic distance is too close or if desired genotypes are not associated with selectable marker or not readily isolated by the marker. Importantly, genetic crossing also mixes epigenetic status of genome. For example, telomere length will change if strains harboring distinct mean length of telomeres are crossed. These risks are avoidable when sequential gene targeting is performed. Therefore, $\mathrm{ura}^{+}{ }^{+} \mathrm{arg}^{+}$and his ${ }^{+}$and LEU2 cassettes are still used, as these auxotrophic markers contain their own distinct promoters and terminators [6-9].

The recently developed genome editing technology, CRISPR (Clustered regularly interspaced short palindromic repeats) and Cas9 (CRISPR-associated protein-9 endonuclease), has become available to use in fission yeast [10]. In this system, the Cas9 nuclease is targeted to specific regions of genomic DNA by its CRISPR RNA component, a so-called guide RNA that binds to the target DNA, resulting in Cas9-mediated DNA double-strand breaks (DSBs) at the target genomic locus. This system does not require selection cassettes, as cells confer constitutive DNA DSBs until the target DNA sequence is lost or altered. Because a selection cassette is not required, this method is ideal for the sequential targeting of multiple genes/loci; in particular for gene disruption and for introducing point mutations or insertions using a designed DNA repair template. However, although the CRISPR/Cas9 strategy is widely used for genetic engineering in animals and humans, the use of this method to mutate genes in fission yeast currently holds a number of limitations. It is often necessary to design a number of guide RNAs, which need to be cloned into a fission yeast CRISPR/Cas9 plasmid [10]. Target sequences must be unique, 20 bases long, and are limited to loci upstream of a protospacer adjacent motif (-NGG for Cas9). This means that the designed DNA repair template will recombine only at the DSB site, and the substitution of the designed point mutation needs to be close to the DSB site [11]. It is also noted that adenine and thymine rich sequences cannot be targeted. Hence, whereas gene disruption, single point mutations and insertions are relatively straightforward for the CRISPR/Cas9 method, replacement of a large genomic DNA region with the designed DNA template, such as wide range multiple mutagenesis of the whole gene sequence [12], is not feasible. Thus, traditional homologous recombination-based genetic engineering remains the most robust method to perform genetic manipulations in fission yeast.

Here, we report optimization of the classical PCR-based targeting method by modification of well-known MX6 markers, which enables sequential knock in/out genes of interest. We also generated the C-terminal tagging vectors for PCR-based gene targeting. In addition, we generated counter selectable drug resistance markers by fusing the herpes simplex virus thymidine kinase (HSV-TK) with antibiotic resistance genes. This can replace the traditionally used counter-selectable $\mathrm{ura}^{+}$cassette. Furthermore, introduction of irreversible mutant loxP (locus of X(cross)-over P1) sites allows recycling of selection cassettes. Finally, using the generated constructs, we report a reliable twostep mutagenesis method, suitable for mutagenesis of essential genes. Altogether, we have demonstrated innovative techniques that address current difficulties in the construction of required fission yeast strains.

\section{Results and discussion}

\section{Cassettes for PCR-based sequential targeting}

Although PCR-based gene targeting by drug selection markers is the conventional approach, it is not efficient if a transforming strain already contains other drug selection markers. All MX6 cassettes share 300 bp of the tef promoter and $200 \mathrm{bp}$ of the tef terminator, which are 
significantly larger than the $80-100$ bp sequences introduced in primers to target genes of interest. Therefore, there is a high chance of recombination between the $M X 6$ cassettes [5]. In order to overcome this problem, CMVneo and CMVzeo cassettes (Life Technology) were cloned to replace the MX6 cassette of the plasmid pFA6a-kanMX6 [2]. Resulting plasmids, pFA6a-neoCV and pFA6a-zeoCV, contain neomycin/kanamycin and Zeocin $^{\mathrm{TM}}$ resistant genes, respectively, flanked by the cytomegalovirus promoter $(P c m v)$ and the simian virus 40 terminator $(T s v 40)$ (Fig. 1a). These cassettes share only 20 bases of the primer annealing sites with the MX6 cassettes, which reduces the risk of unwanted recombination between selection markers. In addition, the tef promoter in the natMX6 cassette, which provides resistance to nourseothricin (Nat), was replaced with the $C M V$ promoter $(P c m v)$, resulting in the plasmid named pFA6a-natCX. The tef terminator of the hygMX6 cassette, which provides resistance to Hygromycin B ( Hyg), was replaced with either the $T s v 40$ terminator or the $S$. cerevisiae LEU2 terminator (Tscleu2), resulting in the plasmids named pFA6a-hygMV and pFA6a-hygML, respectively (Fig. 1a). The $z e o C V$ cassette acts as an equivalent to bleMX6 [4], and provides resistance to Zeocin $^{\mathrm{TM}}$, bleomycin and phleomycin (Fig. 1b). We confirmed that replacement of the promoter or terminator did not impair the function of the drug resistant cassettes. Thus, the newly generated selection cassettes can be used as an alternative to existing MX6 cassettes.

Using the generated cassettes, we tested if they could be used for sequential gene deletion. Two genes, $c r b 2^{+}$and $r d h 54^{+}$, were sequentially targeted in strains bearing both a kanMX6 and natMX6 cassette. Crb2 is the fission yeast ortholog of human 53BP1 [13], and Rdh54 is the equivalent of Rad54, which is required for meiotic recombination [14]. To delete $c r b 2^{+}$, the $h y g M V$ cassette was amplified by PCR using the $c r b 2100 \mathrm{bp}$ primer set (Table 1, crb2 top and bot) and pFA6a-hygMV plasmid as a template. A strain bearing both kanMX6 and natMX6 $\left[h^{90}\right.$ ura4-D18 taz1YFP:kanMX6 sid4-mCherry:natMX6 hht1-Cerulean:ura4 $\left.{ }^{+}\right]$ was transformed with the $c r b 2:: h y g M V$ PCR fragment, and the transformant colonies were selected for resistance to hygromycin B (Hyg). The presence of kanMX6 and natMX6 cassettes was determined by resistance to $\mathrm{G} 418$
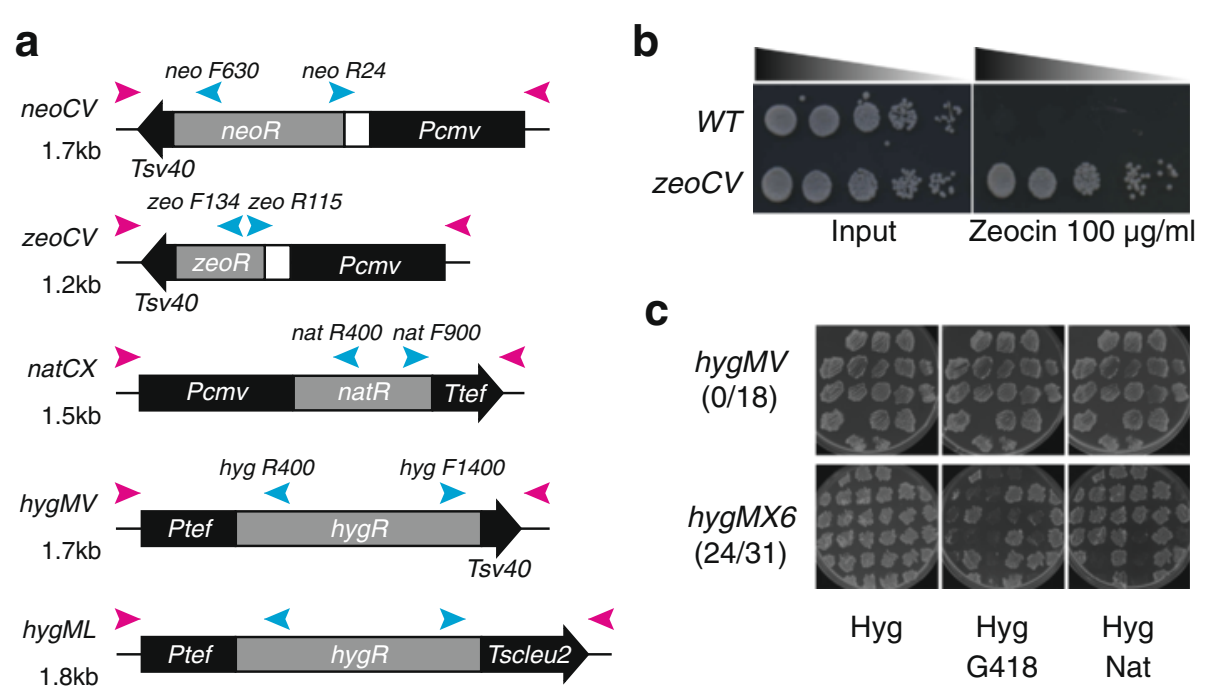

Fig. 1 Selection markers for sequential gene targeting. a Schematic representation of sequential targeting cassettes pFA6a-neoCV, pFA6a-zeoCV, pFA6a-natCX, pFA6a-hygMV and pFA6a-hygML. The region used as a PCR template is shown. The 100 base Top (Tag) primer anneals to the left end (pink arrowhead on the left) and Bot primer anneals to the right end to amplify indicated cassettes (pink arrowheads on the right). The 100 base primers used in this study are listed in Table 1. Cyan arrows represent diagnostic primers used for screening of correct targeting (Table 2). Sequences of primers are shown in Table 2. Backbone vector region (pFA6a) encodes ampicillin resistant cassette and ColE1 bacteria replication origin, and the cassettes were inserted between Pacl and Pmel sites. Black box and black arrow in the cassette represent indicated promoter and terminator, respectively. White box in neoCV and zeoCV indicates the em7 promoter for $E$. coli. The size of the cassette is shown on the left. Transcription direction is toward left for neoCV and zeoCV cassettes and toward right for other cassettes. b Wild-type and rdh54 $\triangle$ cells in which the zeoCV cassette replaced rdh54 were cultured in YES rich media, normalised and serially diluted. Five microliter of diluted fractions were spotted on YES (input) or YES containing $100 \mu \mathrm{g} / \mathrm{ml}$ of Zeocin ${ }^{\mathrm{TM}}$ and incubated at $32^{\circ} \mathrm{C}$ for 3 days. Only cells containing the zeoCV cassette grew on YES with Zeocin. Deletion of the rdh54 gene required for meiosis does not impair cell growth and mitotic DNA damage repair. c Cells carrying kanMX6 or natMX6 cassettes were transformed with hygMV (Top) or hygMX6 (Bottom) and selected for on YES plates containing $100 \mu$ g/ml hygromycin ( $\mathrm{Hyg})$. Eighteen and 31 colonies that were randomly picked for hygMV and hygMX6 transformations respectively, were re-streaked on YES plates containing $100 \mu \mathrm{g} / \mathrm{ml} \mathrm{Hyg} \mathrm{(Left} \mathrm{panel).} \mathrm{Cells} \mathrm{were} \mathrm{then} \mathrm{replica-plated} \mathrm{to} \mathrm{YES} \mathrm{plates} \mathrm{containing} 100 \mathrm{\mu g} / \mathrm{ml} \mathrm{Hyg}$ plus either $100 \mathrm{\mu g} /$ $\mathrm{ml} \mathrm{G418} \mathrm{(Middle} \mathrm{panel)} \mathrm{or} 100 \mu \mathrm{g} / \mathrm{ml}$ ClonNat (Nat) (Right panel). In case of hygMV transformation, all transformed cells retained resistance to G418 and ClonNat, whereas in case of hygMX6, only seven out of 31 did 
Table 1 One hundred base primers for PCR-based targeting fragments used in this study

\begin{tabular}{|c|c|}
\hline Name & Sequence \\
\hline crb2 Top & $\begin{array}{l}\text { CCCTGGTTAAATTGTAGTTCTGACAATTG } \\
\text { TGAGGTATTITAGATGTITCAATATTTGT } \\
\text { TGGAAAGTITAACAATATTCGGATCCCCG } \\
\text { GGTAATAA }\end{array}$ \\
\hline crb2 Bot & $\begin{array}{l}\text { CTAAAATTAATAAAAAGCTAAATTAATGA } \\
\text { GAGTGAAACTCAGGGGGAGTTAGTAAAA } \\
\text { ATAACTATATCAAAAAACCAAAAGAATTC } \\
\text { GAGCTCGTTTAAAC }\end{array}$ \\
\hline rdh54 Top & $\begin{array}{l}\text { ACTTGAACACCACACGTTCGGTCTCATA } \\
\text { GTATTGTTGAGTAAATAAACACAGTTAC } \\
\text { CAAAGAGAATTGAACCTACTTTCGGA } \\
\text { TCCCCGGGTAATTAA }\end{array}$ \\
\hline rdh54 Bot & $\begin{array}{l}\text { TGCTCATAGAGATTGCCCAGTATCGAAG } \\
\text { GCTTGCTCAAAAGCGTTGTTTCTAATGGG } \\
\text { CAAGGAAAAACCGCTTACGCCTCGAATT } \\
\text { CGAGCTCGTITAAAC }\end{array}$ \\
\hline tpzl Tag & $\begin{array}{l}\text { CTCTGAGGCCTGTGAAATGTGTCGGCTTG } \\
\text { GGCTACCTCATGGATCATTCTITGAGCTAT } \\
\text { TGCGAGATTGGAAAAAAATAGAGGAGTT } \\
\text { TCGAAACAAAAGCCGGATCCCCGGGTA } \\
\text { ATTAA }\end{array}$ \\
\hline tpz1 Bot & $\begin{array}{l}\text { TTGGTCCGTTGTAAGCCATTTCACTGTATGT } \\
\text { CTGTAACAGTTAACTCCGTACTTAGTAAA } \\
\text { ATGTTAGTAAAAAAGGAAGATATGTGATA } \\
\text { CAGCAATTGAGAATTCGAGCTCGTTTAAAC }\end{array}$ \\
\hline tpzl Top & $\begin{array}{l}\text { ATCAACAGACTTCAGTCAGCACTGTTACT } \\
\text { TATTAAAAAAAGTTGATIITATATAAAA } \\
\text { GTTAGCTGCGTTAAACAGTGCACGGATC } \\
\text { CCCGGGTTAATTAA }\end{array}$ \\
\hline
\end{tabular}

Under line encodes annealing site for the template. Top primer contains CGGATCCC CGGGTTAATTAA at the $3^{\prime}$ end and 80-100 base upstream sequence of the target site at the $5^{\prime}$ end. Bot primer contains GAATTCGAGCTCGTTAAAC at the $3^{\prime}$ end and $80-100$ base downstream complement sequence of the target site at the $5^{\prime}$ end

and ClonNat (Nat), respectively. The hygMV cassette was therefore successfully integrated into the genome without recombining with the MX6 cassettes present, conferring resistance to multiple antibiotics (Fig. 1c: hygMV panel). Deletion of crb2 by hygMV was confirmed by diagnostic PCR using a forward primer within the $h y g^{R}$ gene and a reverse primer downstream of crb2 (for sequences see Table 2, hyg $F 1400$ and $c r b 2$ R280D). In contrast, transformation with the $c r b 2:: h y g M X 6$ PCR fragment resulted in replacement of kanMX or natMX6 markers, assessed by gain of sensitivity to G418 and Nat, respectively (Fig. 1c: hygMX6 panel). Next, the $r d h 54^{+}$gene was deleted in a similar fashion, using a $r d h 54:: z e o C V$ fragment generated by PCR using the $r d h 54100 b p$ primer set and pFA6a-zeoCV plasmid as a template. Like $h y g M V$, zeoCV did not recombine with any of existent marker cassettes (data not shown). The $r d h 54$ deletion in the resulting strain $\left[h^{90}\right.$ ura4-D18 crb2::hygMV rdh54::zeoCV taz1-YFP:kanMX6 sid4-mCherry:natMX6 hht1-Cerulean:ura4 $\left.{ }^{+}\right]$was confirmed by diagnostic PCR using primers $r d h 54$ R265D and zeo R115 (Table 2). We also confirmed that neoCV, hygML and natCX did not
Table 2 Primers for diagnostic PCR

\begin{tabular}{|c|c|}
\hline Name & Sequence \\
\hline neo R24 & GTGCAATCCATCTTGTTCAATC \\
\hline neo F630 & TTCTGGATTCATCGACTGTGG \\
\hline zeo $R 115$ & CGAAGTCGTCCTCCACGAAG \\
\hline zeo F134 & ACGTGACCCTGTTCATCAGC \\
\hline nat F900 & GGGGTTCACCCTCTGCGGCC \\
\hline nat $R 400$ & GGGACACTGGTGCGGTACCG \\
\hline hyg F1400 & CCGTCTGGACCGATGGCTGT \\
\hline hyg $R 400$ & CTTCTCGACAGACGTCGCGG \\
\hline kan F800 & GGATTCAGTCGTCACTCATGGTG \\
\hline kan $R 276$ & ATGCATCATCAGGAGTACGG \\
\hline TK F1040 & TACCGACGATCTGCGACCTG \\
\hline TK R90 & ACTTCCGTGGCTTCTTGCTG \\
\hline adh1 R540U & AGGAATACGGATACGATGGAG \\
\hline Ptef R81 & ACATGGGGATGTATGGGCTA \\
\hline Ttef F1 & CAGTACTGACAATAAAAAGATTCTTG \\
\hline crb2 R280D & CAACCATTCGAAACCTGCTAC \\
\hline $\operatorname{rdh} 54$ R265D & GAAAGCCAGAAACAGACAAGC \\
\hline $\operatorname{tpz1} F 842 U$ & GGTAGTAGGCTAAATGTGAGTTG \\
\hline $\operatorname{tpzl} R 400 D$ & CCCTTAGAAGATAAGCTCAACC \\
\hline
\end{tabular}

recombine with the MX6 cassettes (data not shown). Therefore, we conclude that replacement of the tef promoter and/or terminator sequences prevents recombination of newly introduced drug selection cassette with the MX6 cassettes already existing in a strain.

\section{HSV-TK chimera selection markers}

Until recently, the $\mathrm{ura}^{+}$auxotrophic marker was the only marker in the fission yeast system to be both selectable and counter-selectable. Cells carrying $\mathrm{ura}^{+}$gene can be eliminated by growing on media supplemented with 5-Fluoroorotic acid (5-FOA), as Ura4 converts 5FOA to toxic fluorodeoxyuridine [7]. Alternatively, the thymidine kinase from herpes simplex virus (HSV-TK, or TK) is available for use as a negative selection marker [15]. TK phosphorylates 5-Fluoro-2'-deoxyuridine (FdU, Floxuridin or FUDR) to give 5-Fluoro-2'-deoxyuridine-5monophosphate (FdUMP). FdUMP blocks activity of thymidylate synthase, resulting in inhibition of 2'-deoxythymidine-5-triphosphate (dTTP) synthesis, leading to loss of the dTTP pool and cell death. Hence, strains expressing a $T K$ cassette cannot grow in the presence of FdU $[15,16]$. Unlike the $\mathrm{ura}^{+}$cassette, the TK cassette can be used with any genotype background. Although fission yeast carrying the $T K$ cassette are sensitive to FdU, a positive selectable marker is also needed in order to isolate cells carrying $T K$ [15]. This issue was solved by 
a fusion of the $T K$ gene with a positive selectable marker in mammalian systems $[17,18]$.

We decided to create fused selection markers for fission yeast, which express fusion antibiotic resistance genes with the $T K$ gene that serve for both positive and negative selection by chemical treatments. We first inserted the TK gene upstream of the gene coding sequences in kanMX6 and natMX6, giving TKanMX6 and TKnatMX6. To assess function of the fusion genes, the TK-fusion cassettes were inserted at the $l e u 1^{+}$locus. As expected, cells carrying TKanMX6 and TKnatMX6 were resistant to G418 and
Nat, respectively. However, these cells grew in the presence of $100 \mu \mathrm{g} / \mathrm{ml} \mathrm{FdU,} \mathrm{suggesting} \mathrm{that} \mathrm{the} \mathrm{fused} T K$ gene is not functional (see the TKnatMX6 in Fig. 2b). We speculated that the level of TK, expressed by the tef promoter, was not sufficient to convert FdU for a toxic amount of FdUMP, as previously published the $T K$ cassette for fission yeast uses the stronger adh1 promoter [15]. We therefore replaced the tef promoter with a stronger $C M V$ promoter (giving TKnatCX, Fig. 2a), which indeed enhanced cells sensitivity to FdU (Fig. 2b). Furthermore, an even greater sensitivity was obtained with replacement of the $S$. pombe adh1

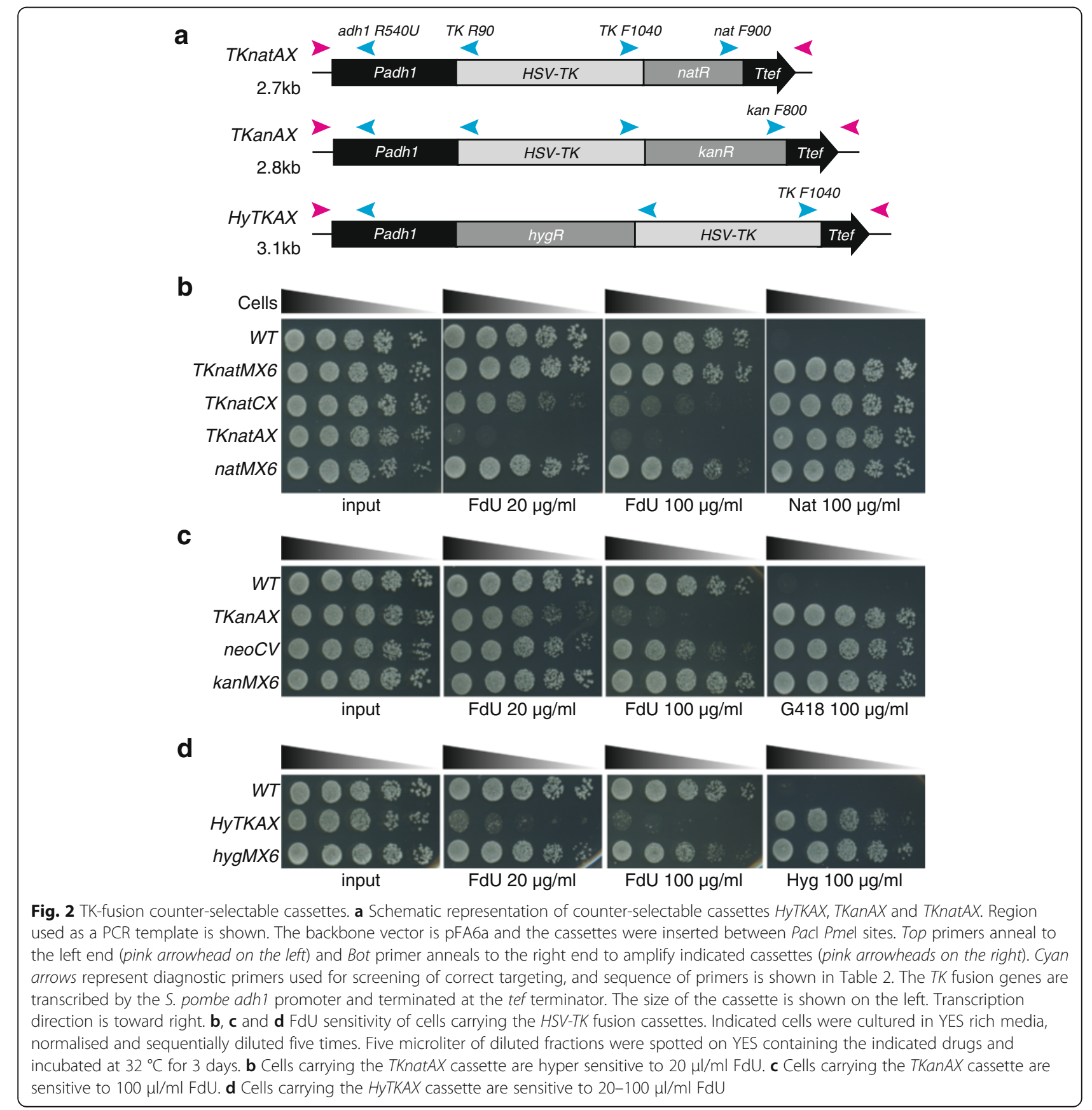


promoter, giving TKnatAX (Fig. 2a and b). The success of this finding was reiterated with the $T K-\mathrm{kan}^{R}$ fusion gene, giving TKanAX (Fig. 2a). Cells carrying TKanAX were fully resistant to G418 and displayed an intermediate sensitivity to FdU (Fig. 2c). Finally, HSV-TK was inserted after the $h y g^{R}$ gene coding sequence, similar to the HyTK or HygRTK fusion genes used in mammalian systems [17], giving HyTKAX (Fig. 2a). Cells carrying HyTKAX were resistant to hygromycin B and sensitive to FdU (Fig. 2d). In conclusion, fusions of the yeast antibiotic markers with the $T K$ gene are functional, allowing both positive and negative selections of cells carrying the fusion cassettes.

In yeast, low sensitivity to FdU is partially caused by poor uptake of thymidine. This problem can be overcome by ectopic expression of human equilibrative nucleoside transporter (ENT), which is highly efficient at the uptake of thymidine analogs [19]. However, this strategy requires cells to carry the ENT expression cassette. Toxicity of FdU in the presence of TK is caused by impaired deoxythymidine5-monophosphate (dTMP) synthesis and reduced dTTP levels [16]. Hence, we anticipated that reduction of the deoxynucleotide (dNTP) pool by hydroxyurea (HU) might further reduce the dTTP level, leading to increased sensitivity to FdU. To test this possibility, cells carrying TKnatAX, TKanAX and HyTKAX were spotted on YES media containing increasing concentrations of $\mathrm{HU}(1,2.5$ and $5 \mathrm{mM})$ along with $100 \mu \mathrm{g} / \mathrm{ml} \mathrm{FdU} \mathrm{(Fig.} \mathrm{3).} \mathrm{The} \mathrm{growth} \mathrm{of} \mathrm{cells}$ carrying TKanAX was significantly suppressed by FdU in the presence of $\mathrm{HU}$, as represented by microcolonies. Taken together, we recommend the following concentrations for FdU anti-selections: $20 \mu \mathrm{g} / \mathrm{ml} \mathrm{FdU} \mathrm{for} \mathrm{TKnatAX,} 100 \mu \mathrm{g} / \mathrm{ml}$ FdU for HyTKAX and $100 \mu \mathrm{g} / \mathrm{ml}$ FdU plus $5 \mathrm{mM}$ HU for TKanAX.

\section{Mutants defective in DNA replication checkpoint and homologous recombination are sensitive to FdU}

Although the $T K$ cassette is a useful tool, we have come across a number of fission yeast mutants that exhibited sensitivity to FdU while not containing the TK cassette. Specifically, our data suggest that mutants defective in DNA replication and DNA damage response pathways are sensitive to FdU. It has previously been reported that rad3 $\Delta$ cells, which lack the DNA damage checkpoint protein Rad3 (a fission yeast ATR homolog [20]), are sensitive to the deoxythymidine variant, 5'-Ethyl-2'-deoxyuridine in the presence of TK [21]. Surprisingly, we identified that rad3 $\Delta$ cells that did not express TK were also sensitive to $20 \mu \mathrm{g} / \mathrm{ml}$ FdU (Fig. 4a). In response to DNA damage and DNA replication problems, Rad3 activates Chk1 and Cds1, respectively [20]. Interestingly, $c d s 1 \Delta$ cells were sensitive to $100 \mu \mathrm{g} / \mathrm{ml} \mathrm{FdU}$, whereas chk1 $\Delta$ cells were only mildly sensitive to FdU (Fig. 4b). Moreover, homologous recombination

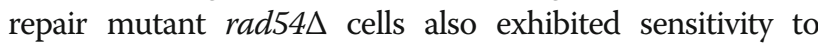
$100 \mu \mathrm{g} / \mathrm{ml}$ FdU (Fig. 4b). Whilst Rad54 is essential for homologous recombination, the Rad55-Rad57 complex acts in parallel to Swi5-Sfr1 and upstream of Rad54 [22]. As such, rad55 $\Delta$ cells exhibited only mild sensitivity. Although it remains unclear how FdU can be converted to FdUMP under DNA replication stress, our data suggest that mutants defective in DNA replication and DNA damage response pathways are sensitive to FdU. Therefore, we recommend to test working strains for the sensitivity to FdU prior to performing counter-selection screening.

\section{The Cre/loxp system can be utilized for the recycling of counter-selectable markers}

Another way to address the limited availability of selection markers is to recycle the cassette using the Cre/loxP approach [23-25]. In this system, Cre recombinase recombines two short loxP sequence sites to generate crossover products. Hence, a loxP-flanked (floxed) gene of interest or selection marker can be deleted via expression of Cre recombinase. To generate conditional pop-out markers for TKanAX, TKnatAX and HyTKAX, mutant variants of loxP sequences - lox71 and lox66 - were introduced at the start and end of the cassettes. Resulting cassettes were named

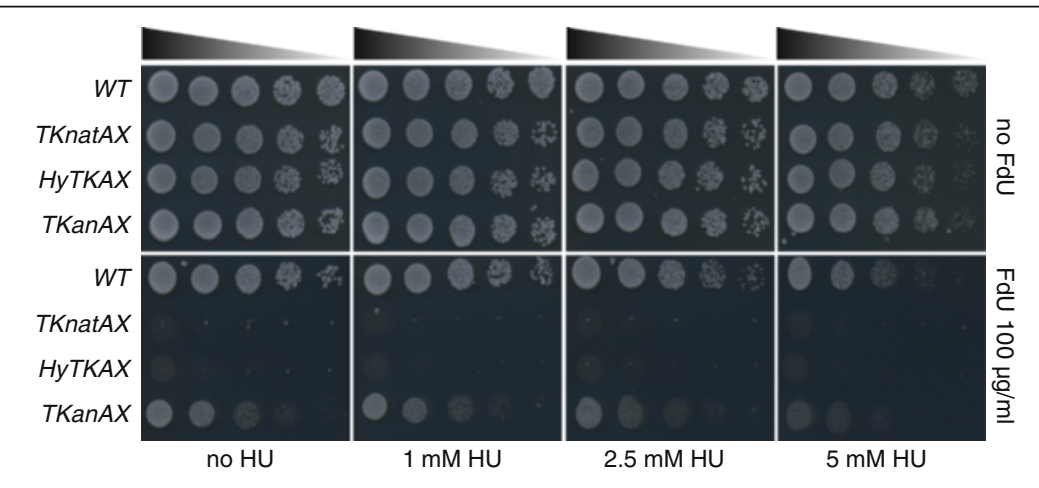

Fig. 3 Concomitant treatment with HU enhances sensitivity to FdU. Indicated cells were cultured in YES, normalised and serially diluted five times with YES. Five microliter of diluted fractions were spotted on rich media containing the indicated drugs and incubated at $32^{\circ} \mathrm{C}$ for 3 days. Cells carrying TKanAX grew poorly in YES containing $5 \mathrm{mM} \mathrm{HU}$ and $100 \mu \mathrm{g} / \mathrm{ml} \mathrm{FdU}$ 


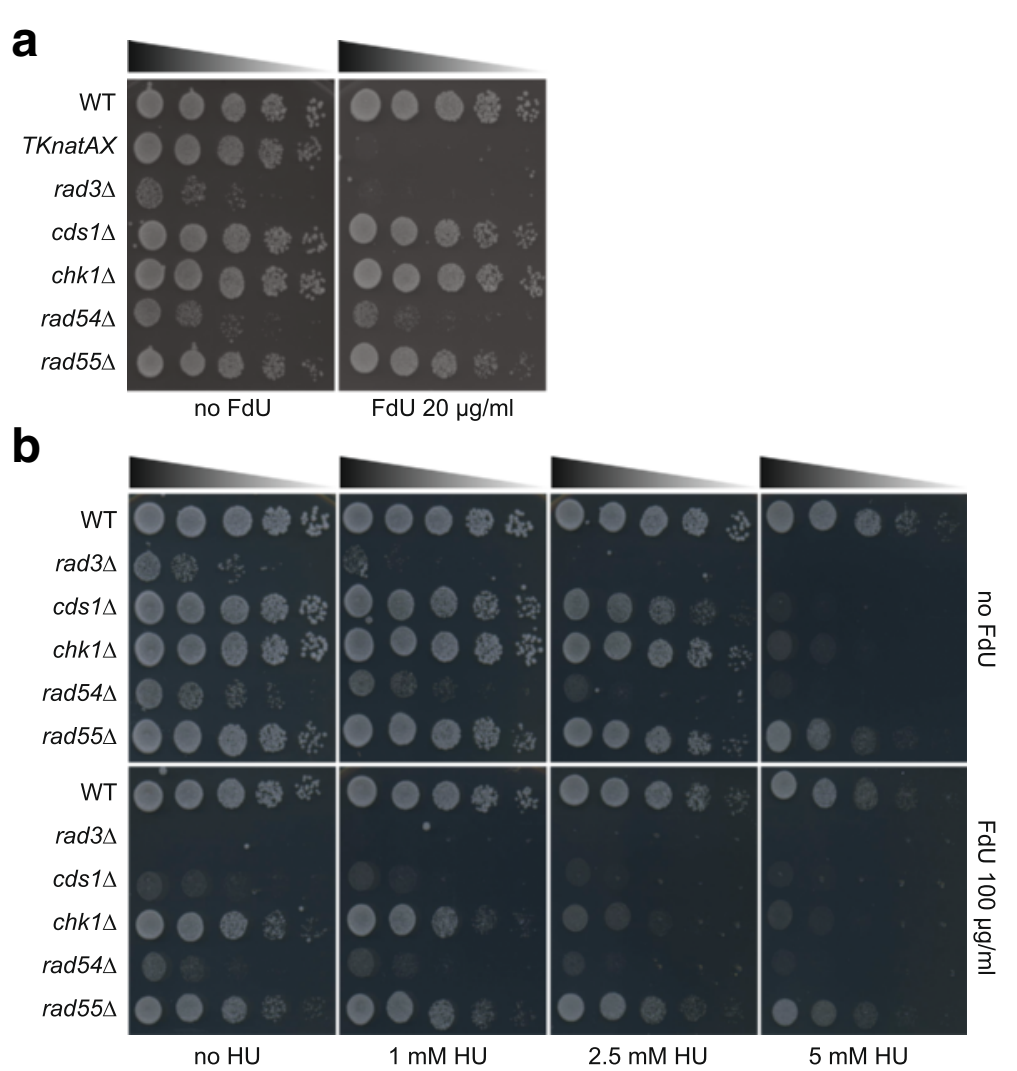

Fig. $4 \mathrm{rad} 3 \Delta, c d s 1 \triangle$ and rad54 $\triangle$ are sensitive to FdU. Indicated cells were cultured in YES, normalised and 5 times sequentially diluted with YES. Five microliter of diluted fractions were spotted on rich media containing the indicated drugs and incubated at $32^{\circ} \mathrm{C}$ for 3 days. a rad $3 \Delta$ cells are hyper


compared to wild type cells

FTKanAX, FTKnatAX and FHyTKAX, respectively (Fig. 5a, Table 3). Although the lox71 and lox66 sequences are substrates for Cre recombinase, the crossover results in the generation of wild type loxP and double mutated loxP sequences; the latter is no longer recognized by the recombinase [26]. Therefore, the scar of a lox71-lox66 recombined site will not crossover with a newly introduced loxPcontaining marker in the genome.

We also generated a series of the Cre expression vectors, encoding a fusion protein, comprised of the three tandem hemagglutinin (HA) genes, a nuclear localization signal (nls) sequence and Cre recombinase (3xHA-nls-Cre), under the control of a $C M V$ promoter (Fig. $5 \mathrm{~b}$ ). To maximize versatility, the Cre expression vector carries a series of the selection markers; kanMX6, TKkanAX, hygMX6, HyTKAX, natMX6, TKnatAX or the aur $r^{R}$ cassette, which encodes gene resistance to aureobasidin A [27], as well as auxotrophic markers, including $\mathrm{ura}^{+}, \mathrm{arg}^{+}, \mathrm{leu} 1^{+}$and ade $^{+}$(Table 4). Altogether, a choice of three 'floxed' markers and a series of the Cre expression vectors would maximize the utility for genome engineering.

Using the Cre expression vector, the efficiency of the FTKnatAX cassette removal was assessed. Cells carrying the FTKnatAX cassette were transformed with the Cre expression vector, and were directly plated on FdU plates. Total 162 colonies were formed on the FdU plate after Cre expression, and all were lost the resistance to Nat. Ten clones were randomly selected and their loss of the FTKnatAX cassette was confirmed by PCR. Thus, FdU counter selection along with Cre expression efficiently eliminates cells retaining the FTKnatAX Cassette.

Tandem epitopes C-terminal tagging plasmids, pNX3 series In addition to creating a series of plasmids useful for the sequential modification of genes, we have also created similar plasmids for $\mathrm{C}$-terminal tagging of gene products. Tandem repeats of epitope tags enhance the detection efficiency of target proteins, which is important for their visualization, especially if they have low-abundance. However, larger tags can potentially interfere with protein function. Therefore, the size of the tag needs to be optimized for each protein of interest. To address this, three tandem genes coding for either HA, PK (V5) and FLAG epitopes, flanked by $N h e \mathrm{I}$ and $\mathrm{XbaI}$ restriction sites were synthesized, and cloned between PacI and AscI sites of the pFA6a-kanMX6 C-terminal tagging plasmid [2] (Fig. 6a 

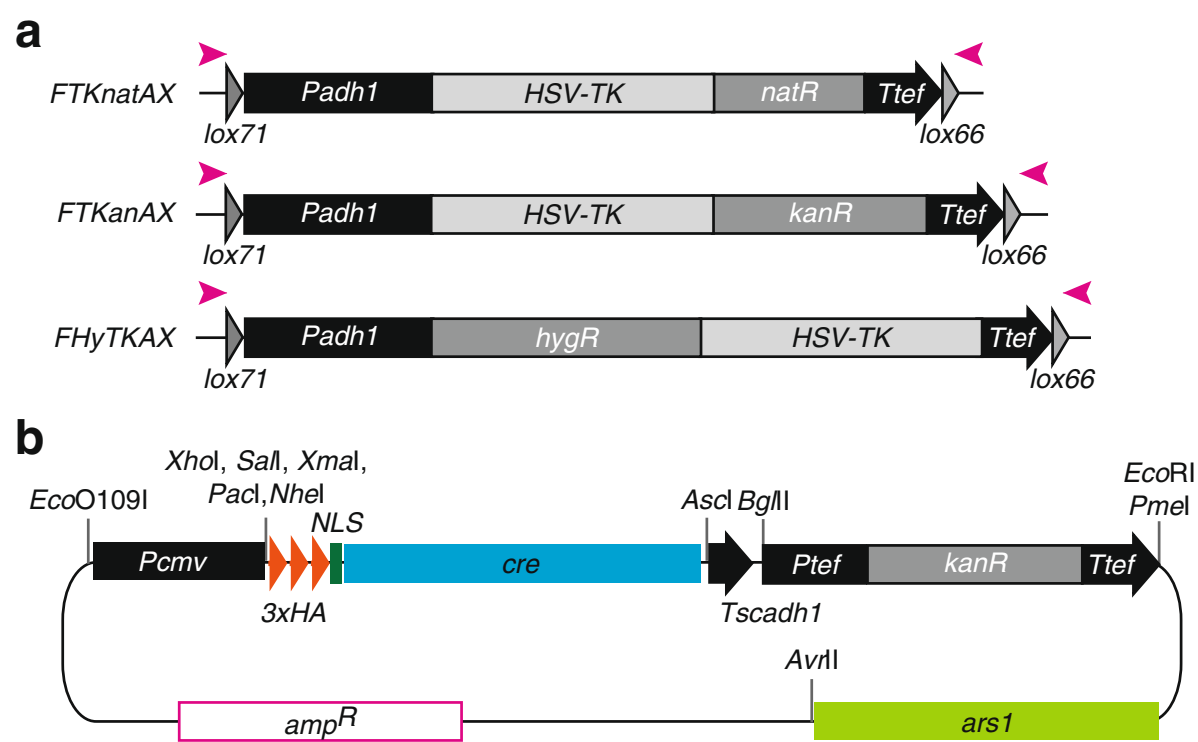

Fig. 5 Cre expression vector and loxP cassette. a Schematic representation of loxP-flanked (floxed) TK-fusion cassettes FHyTKAX, FTKanAX and FTKnatAX. Region used as a PCR template is shown. Forward direction of lox71 and lox66 sequences are inserted into the TK cassettes shown in Fig. 2a. b Schematic representation of pNXRVa-HACre. Fusion protein of three tandem HA epitope tag, NLS (nuclear localization signal) and Cre recombinase is expressed by the constitutively active CMV promoter. kanMX6 is inserted between Bglll and Pmel sites. The genomic DNA fragment containing the early replication origin ARS1 is inserted downstream of the kanMX6 cassette. A number of unique restriction enzyme sites are indicated

and b). PCR-based amplification of the C-terminal tagging plasmid for gene targeting results in addition of a peptide - Arg-Ile-Pro-Gly-Leu-Ile-Asn-Ala-Ser - which acts as a linker between the target protein and the epitopes (Fig. 6A). The resulting plasmids were named pNX3 (plasmid NheI-XbaI version three).

The pNX3 plasmid series was extended by replacing kanMX6 with other drug resistance cassettes [hygMX6,

Table 3 The targeting cassette vectors

\begin{tabular}{|c|c|c|}
\hline Name & Promoter/gene/terminator & Selection/counter \\
\hline pFA6a-kanMX6 & Ptef/kans/Ttef & G418 \\
\hline pFA6a-neoCV & Pcmv/neo ${ }^{R} / T s v 40$ & G418 \\
\hline pFA6a-zeoCV & Pcmv/zeo ${ }^{R} / T_{s v} 40$ & Zeocin \\
\hline pFA6a-hygMX6 & Ptef/hph/Ttef & G418 \\
\hline pFA6a-hygMV & Ptef/hph/Tsv40 & Hygromycin B \\
\hline pFA6a-hygML & Ptef/hph/Tscleu2 & Hygromycin B \\
\hline pFA6a-natMX6 & Ptef/nat ${ }^{R} /$ tef & ClonNat \\
\hline pFA6a-natCX & Pcmv/nat ${ }^{R} /$ tef & ClonNat \\
\hline pFA6a-TKnatCX & Pcmv/TK-nat ${ }^{R} /$ tef & ClonNat/FdU \\
\hline pFA6a-TKnatAX & Padh/TK-nat $t^{R} /$ tef & ClonNat/FdU \\
\hline pFA6a-TKanAX & Padh/TK-kan ${ }^{R} /$ tef & $\mathrm{G} 418 / \mathrm{FdU}+\mathrm{HU}$ \\
\hline pFA6a-HyTKAX & Padh/hph-TK/tef & Hygromycin $\mathrm{B} / \mathrm{FdU}$ \\
\hline pFA6a-FTKnatAX & lox71-Padh/TK-nat ${ }^{R} /$ Ttef-lox66 & ClonNat/FdU \\
\hline pFA6a-FTKanAX & lox71-Padh/TK-kan ${ }^{R} /$ tef-lox66 & $\mathrm{G} 418 / \mathrm{FdU}+\mathrm{HU}$ \\
\hline pFA6a-FHyTKAX & lox71-Padh/hph-TK/tef-lox66 & Hygromycin B/FdU \\
\hline
\end{tabular}

natMX6, neoCV, hygML, natCX and zeoCV] or $\mathrm{ura}^{+}$cassette. The cleavage site of NheI is compatible with that of $X b a \mathrm{I}$, which allows ligation of epitope tags. For example, $3 x \mathrm{XK}$ can be sequentially subcloned to generate $6 \mathrm{xPK}$, 9xPK and 12xPK-tagging plasmids. A list of the pNX3 plasmid series created is shown in Table 5. All plasmids were verified by DNA sequencing, and some of the constructs have been used elsewhere [12, 28].

Using PK-tagging plasmids from this series and the tpz1 Tag and Bot 100 base primer set, we successfully inserted the gene encoding three, six, nine and 12 tandem-repeats of the PK epitope at the end of the coding gene of $t p z 1^{+}$to endogenously express Tpz1 with the PK tagged at the Cterminus (Fig. 6c). Tpz1 is a component of telomere shelterin complex and is a homolog of human TPP1. Tpz1 both positively and negatively regulates telomere length by interacting with different factors [28, 29]. One interacting partner binds to the C-terminal ends of Tpzl, which is required for formation of shelterin to suppress telomere elongation [29-31]. Deletion of tpz1 leads to telomere deprotection and cell death [31]. As expected, the efficiency of detection of Tpz1 was increased with a number of PK repeats, and the observed difference in band sizes was due to a different number of $3 \times \mathrm{PK}$ repeats in each sample (molecular weight of the $3 \mathrm{xPK}$ is $4.2 \mathrm{kDa}$ ). The slower migrating band in each lane, that presumably corresponds to SUMOylated Tpz1 [32], also became readily detectable (Fig. 6c). To determine whether the C-terminal fusion of PK-tags impaired the activity of Tpz1, 
Table 4 The Cre-expression vectors

\begin{tabular}{lll}
\hline Name & Marker cassette & Selection \\
\hline pNXRVa-HACre & kanMX6 & G418 \\
pNXRVat-HACre & TKanAX & G418 \\
pNXRVb-HACre & hygMX6 & Hygromycin B \\
pNXRVbt-HACre & HyTKAX & Hygromycin B \\
pNXRVc-HACre & natMX6 & ClonNat \\
pNXRVct-HACre & TKnatAX $^{+}$ & ClonNat \\
pNXRVd-HACre & urat $^{+}$ & -uracil \\
pNXRVg-HACre & arg3 $^{+}$ & -arginine \\
pNXRVh-HACre & leu1 $^{+}$ & -leucin \\
pNXRVj-HACre & aur $^{R}$ & Aureobasidin A \\
pNXRVk-HACre & ade6 $^{+}$ & -adenine \\
\hline
\end{tabular}

we measured telomere length of cells bearing Tpz1 fused to a different number of repeats of PK epitopes (Fig. 6d). Whereas three and six tandem PK tags did not affect telomere length homeostasis, nine tandem PK tags led to slightly elongated telomeres. Thus, we conclude that, although larger tags make proteins and their modifications more visible, they may increase the risk of interfering with function.

\section{Two-step transformation for gene editing at the endogenous locus}

A traditional two-step method can utilize the $u r a 4^{+}$ cassette to introduce point mutations [7]. First, the gene of interest is replaced by the $\mathrm{ura}^{+}$cassette in a strain where the endogenous $\mathrm{ura}^{+}$gene region has been deleted (ura4-D18). Next, the inserted ura4 $^{+}$cassette is replaced with the mutagenized gene allele. The successfully transformed cells, which lost $\mathrm{ura4}^{+}$, are initially selected using 5-FOA. We previously utilized this method with success to mutagenize the rap1 gene [12]. This strategy requires the ura4-D18 mutation in the strain and further epitope tagging may be necessary to determine the stability of the mutant product downstream. To mutagenize essential genes, a diploid strain should be used in which only one allele of the target gene is replaced with the $\mathrm{ura}^{+}$cassette. However, because the wild type allele has better homology, the targeting construct favors the wild type allele over the deleted allele. Thus, although treatment with 5-FOA can reduce occurrence of false positives, the presence of the wild type allele makes difficult to target the $\mathrm{ura}^{+}$ replaced locus in diploid cells.

To address this issue, we propose a new method, which utilizes anti-selection markers. In this method, the gene of interest is replaced with a negative selection cassette from the TK-fusion series. The second step uses a plasmid from the pNX3 series to introduce the desired modification to the gene. Briefly, the gene of interest together with its endogenous promoter is subcloned into a pNX3 plasmid and mutagenized as desired. The TKfusion cassettes contain the tef terminator but not the tef promoter. The shared homology between the tef terminator and the promoter region of the gene in the pNX3 plasmid and the TKnat/kan cassettes permits insertion of the modified gene at the deleted allele via homology directed repair.

As an example, we utilized this method to introduce point mutations in the $t p z 1^{+}$gene. As $t p z 1^{+}$is essential gene, we first deleted one tpz1 allele in a diploid strain using the PCR-based TKnatCX recombination method (Fig. 7a). The cloned $t p z 1^{+}$in the pNX3a-3HA plasmid (pNX3a-tpz1-HA3) was mutagenized at the position c.322-323 to give the mutation K75A. The resulting construct, pNX3a-tpz1-K75A-HA3, was digested with the restriction enzymes, and the tpzl heterozygous diploid strain was transformed with this targeting construct (Fig. 7b). The resulting transformants were selected by G418 resistance. Most of the positive colonies lost their Nat resistance as successful insertion of the mutant gene would result in removal of the TKnatCX cassette. In our experience, success rate of the gene replacement was $96 \%$ (25 cells lost the Nat resistance out of 26 cells resistant to G418). Diagnostic PCR using primer sets shown in Fig. 7c confirmed that all of the transfomants that lost Nat resistance had the correct replacement of tpz1(K75A)-3xHA:kanMX6 at the TKnatCM site. Expression of Tpz1(K75A)-3xHA was confirmed by Western blot. Finally, the resulting heterozygous diploid strain $\left(h^{-} / h^{+}\right.$ade6-M210/M216 tpz1(K75A)-3xHA:kanMX6/+) was starved to induce sporulation, and the offspring was selected on the G418 to isolate a $\operatorname{tpz1}(\mathrm{K} 75 \mathrm{~A})$ haploid strain. Expression level, function of Tpz1(K75A), and the phenotype of this mutant strain were reported previously [28]. Therefore we showed that this method can be successfully and reliably used to target and modify genes to give stable products.

\section{Conclusions}

We have demonstrated that the replacement of promoter and/or terminator sequences in drug resistance cassettes can eliminate the need to replace existing markers in working strains. This enables sequential genetic manipulations to be performed without making changes to the genetic and epigenetic background of strains. In addition, we have shown that the TK-fusion markers can be used to replace genes and subsequently swapped in order to counter-select for undesirable genotypes. While PCR-based gene targeting remains a powerful and cost-effective method, the modified plasmids and optimized techniques presented in this study would enable researchers to perform their desired genetic manipulations more efficiently and reliably. 


\section{a}

Three tandem HA epitope tag sequence (HA3) between Sall and Ascl

NotI PvuII BsiwI Sali BamHI XmaI PacI NheI

acgeggecgccagctgaagcttegtacgctgcaggtcga CGG ATC CCC GGG TTA ATT AAC GCT AGC ATG TAC CCA TAC GAT GTT CCT GAC TAT GCG GGC Arg Ile Pro Gly Leu Ile Asn Ala Ser Met Tyr Pro Tyr Asp Val Pro Asp Tyr Ala Gly

TAC CCG TAT GAC GTG CCG GAC TAT GCA GGA GCC TAT CCT TAT GAC GTT CCA GAT TAC GCT GGT XCT AGA TGA ggcgcgce

TAC CCG TAT GAC GTG CCG GAC TAT GCA GGA GCC TAT CCT TAT GAC GTT CCA GAT TAC GCT GGT TCT AGA TGA
Tyr Pro Tyr Asp Val Pro Asp Tyr Ala Gly Ala Tyr Pro Tyr Asp Val Pro Asp Tyr Ala Gly Ser Arg *

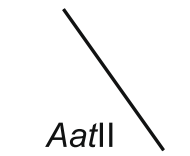

EcoO109I Ndel Notl

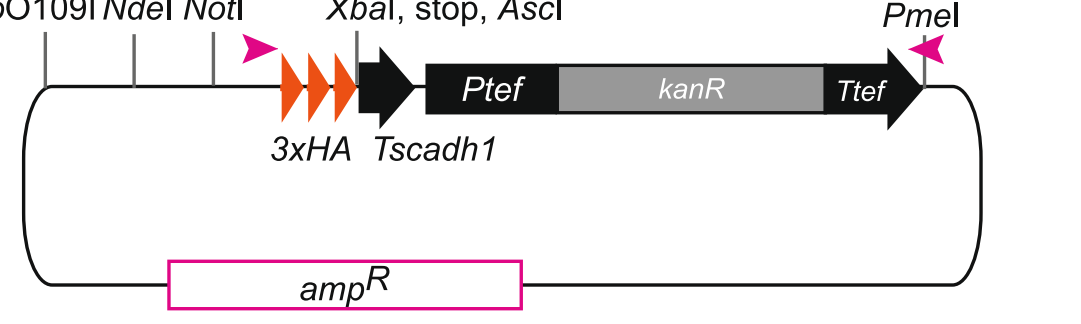

EcoRI

Sacl

b

Three tandem PK epitop tag (PK3) sequence

NheI
GCT AGC GGT AAG CCT ATT CCT AAC CCT CTT TTG GGT CTC GAT TCT ACA

Ala Ser gly Lys Pro Ile Pro Asn Pro Leu Leu Gly Leu Asp Ser Thr

GGT GGA AAA CCA ATC CCC AAC CCA CTC CTC GGC CTT GAC TCA ACT

Gly Gly Lys Pro Ile Pro Asn Pro Leu Leu Gly Leu Asp Ser Thr

GGC AAA CCC ATA CCA AAT CCT TTA CTT GGA TTA GAT AGT ACC TCT AGA

Gly Lys Pro Ile Pro Asn Pro Leu Leu Gly Leu Asp Ser Thr Ser Arg

\section{Three tandem Flag epitop tag (FL3) sequence}

NheI

GCT AGC ATG GGT GAC TAC AAG GAC GAC GAT GAC AAG GGA

Ala Ser Met Gly Asp Tyr Lys Asp Asp Asp Asp Lys Gly

GAT TAC AAA GAT GAC GAC GAT AAG GAC TAC AAG GAC GAC GAT GAC AAG XbaI

GAT TAC AAA GAT GAC GAC GAT AAG GAC TAC AAG GAC GAC GAT GAC AAG TCT AGA
Asp Tyr Lys Asp Asp Asp Asp Lys Asp Tyr Lys Asp Asp Asp Asp Lys Ser Arg

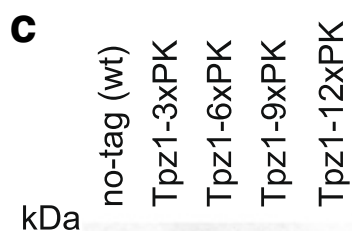

100

75

50

37

25

37

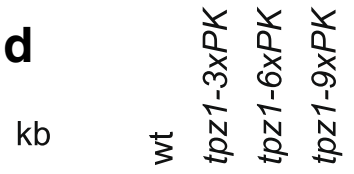

3.0

2.5

2.0

PK

1.5

1.0
0

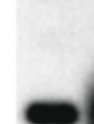

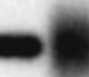
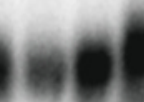

Cdc2

Fig. 6 The $\mathrm{COOH}$-terminus tagging plasmid. a Schematic representation of pNX3a-HA3 and sequence of three tandem HA and multi cloning sites. The HA encoding gene is inserted between Pacl Ascl sites. The 100 base Tag primer anneals to the left end (pink arrowhead on the left in plasmid image and long arrow in HA3 sequence) and Bot primer anneals to the right end to amplify indicated cassettes (pink arrowheads on the right in plasmid image). A number of unique restriction enzyme sites are indicated. $\mathbf{b}$ Sequence of three tandem PK (top) and FLAG (bottom) fragments. pNX3a-PK3 and pNX3a-FL3 plasmids were generated by replacing HA3 sequence between Nhel and Xbal sites in PNX3a-HA3 (a) with indicated PK and FLAG sequences, respectively. c Detection efficiency of PK-tagged Tpz1. Western blot shows detection of PK epitope fused Tpz1. No obvious non-specific bands were detected. Proteins were extracted from cells and subjected to SDS-PAGE. Anti-V5 antibody (AbD Serotec) was used to detect PK fused Tpz1 protein. Anti-Cdc2 antibody (anti-PSTAIRE) (Santa Cruz) was used as a control for loading. d Telomere length homeostasis is slightly impaired with the nine tandem PK tagging of Tpz1. Genomic DNA was harvested from cells cultured over 2 weeks after generation of strains, and digested with EcoRl and separated in $1 \%$ agarose gel. Telomere containing fragments were detected with the synthetic telomeric DNA probe

\section{Methods}

\section{Yeast media}

All media and supplements were purchased from FORMEDIUM $^{\mathrm{T}}$. Fission yeast were grown at $32{ }^{\circ} \mathrm{C}$.

\section{Plasmid constructions}

All plasmids generated are listed in Tables 3, 4 and 5 and are available from Addgene (www.addgene.com). Oligos used for cloning are listed in Table 6. To create pFA6a- 
Table 5 The C-terminal tagging vectors, pNX3 series

\begin{tabular}{|c|c|c|c|}
\hline Name & Epitope tag & Marker cassette & Selection \\
\hline pNX3a-PK3 & $3 \times P K$ & kanMX6 & G418 \\
\hline pNX3a-PK6 & $6 \times P K$ & kanMX6 & G418 \\
\hline pNX3a-PK9 & $9 \times \mathrm{PK}$ & kanMX6 & G418 \\
\hline pNX3a-PK12 & $12 \times P K$ & kanMX6 & G418 \\
\hline pNX3a-FL3 & 3xFlag & kanMX6 & G418 \\
\hline pNX3a-HA3 & $3 \times \mathrm{HA}$ & kanMX6 & G418 \\
\hline pNX3a-Myc13 & $13 x \mathrm{Myc}$ & kanMX6 & G418 \\
\hline pNX3b-PK3 & $3 \times P K$ & hygMX6 & Hygromycin B \\
\hline pNX3b-PK6 & $6 \times \mathrm{PK}$ & hygMX6 & Hygromycin B \\
\hline pNX3b-PK9 & $9 \times \mathrm{PK}$ & hygMX6 & Hygromycin B \\
\hline pNX3b-PK12 & $12 \times P K$ & hygMX6 & Hygromycin B \\
\hline pNX3b-FL3 & 3xFlag & hygMX6 & Hygromycin B \\
\hline pNX3b-HA3 & $3 \times \mathrm{HA}$ & hygMX6 & Hygromycin B \\
\hline pNX3b-Myc13 & 13xMyc & hygMX6 & Hygromycin B \\
\hline pNX3c-PK3 & $3 \times P K$ & natMX6 & CloneNat \\
\hline pNX3c-PK6 & $6 \times P K$ & natMX6 & CloneNat \\
\hline pNX3c-PK9 & $9 \times \mathrm{PK}$ & natMX6 & CloneNat \\
\hline pNX3c-PK12 & $12 \times P K$ & natMX6 & CloneNat \\
\hline pNX3c-FL3 & 3xFlag & natMX6 & CloneNat \\
\hline $\mathrm{pNX} 3 \mathrm{c}-\mathrm{HA} 3$ & $3 \times \mathrm{HA}$ & natMX6 & CloneNat \\
\hline pNX3c-Myc13 & 13xMyc & natMX6 & CloneNat \\
\hline pNX3d-PK3 & $3 \times P K$ & $\mathrm{ura}^{+}$ & -uracil \\
\hline pNX3d-PK6 & $6 \times \mathrm{PK}$ & ura $^{+}$ & -uracil \\
\hline pNX3d-PK9 & $9 \times \mathrm{PK}$ & ura $^{+}$ & -uracil \\
\hline pNX3d-PK12 & $12 \times P K$ & ura $^{+}$ & -uracil \\
\hline pNX3d-FL3 & 3xFlag & $\mathrm{ura}^{+}$ & -uracil \\
\hline pNX3d-HA3 & $3 \times \mathrm{HA}$ & ura $^{+}$ & -uracil \\
\hline pNX3d-Myc13 & 13xMyc & ura $^{+}$ & -uracil \\
\hline pNX3a2-PK3 & $3 \times P K$ & neoCV (rev) & G418 \\
\hline pNX3a2-FL3 & 3xFlag & neoCV (rev) & G418 \\
\hline pNX3a2-HA3 & $3 \times \mathrm{HA}$ & neoCV (rev) & G418 \\
\hline pNX3a2-Myc13 & $13 x$ Myc & neoCV (rev) & G418 \\
\hline pNX3b2-PK3 & $3 \times P K$ & hygML & Hygromycin B \\
\hline pNX3b2-FL3 & 3xFlag & hygML & Hygromycin B \\
\hline pNX3b2-HA3 & $3 \times \mathrm{HA}$ & hygML & Hygromycin B \\
\hline pNX3b2-Myc13 & $13 x$ Myc & hygML & Hygromycin B \\
\hline pNX3c2-PK3 & $3 \times P K$ & natCX & CloneNat \\
\hline $\mathrm{pNX} 3 \mathrm{c}^{-}-\mathrm{HA} 3$ & $3 \times \mathrm{HA}$ & natCX & CloneNat \\
\hline pNX3c2-Myc13 & 13xMyc & natCX & CloneNat \\
\hline pNX3s2-PK3 & $3 \times P K$ & zeoCV (rev) & Zeocin \\
\hline pNX3s2-FL3 & 3xFlag & zeoCV (rev) & Zeocin \\
\hline pNX3s2-HA3 & $3 \times \mathrm{HA}$ & zeoCV (rev) & Zeocin \\
\hline pNX3s2-Myc13 & $13 x$ Myc & zeoCV (rev) & Zeocin \\
\hline
\end{tabular}

(rev): neoCV and zeoCV cassettes are inserted in reverse direction $n e o C V$ and pFA6a-zeoCV plasmids, the CMVneo and $C M V z e o$ cassettes were amplified from pCMVneo and pCMVzeo plasmids, respectively, using primers Pcmv topPmeI and Tsv40 bqt-BglII. The kanMX6 cassette of pFA6akanMX6 was digested with BglII and PmeI and replaced with the CMVneo and CMVzeo cassettes. To create pFA6anatCX, the $C M V$ promoter was amplified from pCMVpuro plasmid using primers Pcmv top-BglII and Tsv40 bot-PmeI, and the tef promoter of the natMX6 cassette in pFA6anatMX6 was digested with BglII and NcoI (blunt ended) and replaced by BglII and KpnI digested (blunt ended) Pcmv PCR fragment. To create pFA6a-hygMV, the terminator of the $s v 40$ was subcloned from pSV2-hyg plasmid. pSV2-hyg was digested with PfMI and HpaI and selfligated to remove the gene encoding large tumor antigen, and the hyg resistant gene with $T s v 40$ was cloned using primers, $\mathrm{Hyg}^{R}$ top-HindIII and Tsv40 bot-PmeI. The tef terminator along with a part of the $h y g$ resistant gene of pFA6a-hygMX6 was replaced with the $h y g^{R}-T s v 40$ PCR product using SacII and PmeI sites, and resulting plasmid was named pFA6a-hygMV. To replace the terminator of the hygMX6 cassette with the S. cerevisiae LEU2 gene, the terminator of LEU2 was cloned from the S. cerevisiae genomic DNA using primers, Tscleu2 top-ClaI-PstI, and Tscleu2 bot-PmeI, and inserted between PstI and ClaI sites of pFA6a. The tef promoter and the hyg resistant gene were cloned using primers, Amp rev and Hyg bot-NheI-ClaI, and inserted at BglII-ClaI sites of pFA6a. The resulting plasmid pFA6a-Ptef-hyg was digested with ClaI and the LEU2 terminator was inserted to generate pFA6a-hygML.

To create pFA6a-TKanMX6 and pFA6a-TKnatMX6, the TK gene was cloned using primers, HSVtk top-SpeI-NcoIHindIII and HSVtk bot-XmaI-NcoI, digested and inserted at NcoI site of pFA6a-kanMX6 and pFA6a-natMX6. To create pFA6a-HyTKMX6, the TK PCR product was digested with $N c o I$ and XmaI (blunt ended) and replaced $n a t^{R}$ gene of pFA6a-natMX6 using NcoI and SphI (blunt ended) sites. The resulting plasmid was named pFA6a-HSVTKMX6. pFA6a-hygML was digested with NheI and PmeI, and Tscleu2 was replaced with the SpeI and PmeI digested HSVTK-Ttef fragment from pFA6a-HSVTKMX6. To create pFA6a-TKnatCX, the $C M V$ promoter was cloned from pCMVzeo using primers Pcmv top-BglII and zeo R115 and digested with $B g l \mathrm{II}$ and $M s c \mathrm{I}$. TKnatMX6 was digested with BglII and SpeI (blunt ended), and the tef promoter was replaced with the $C M V$ promoter.

The adh1 promoter was amplified from S. pombe genomic DNA using primers, Padh1 top-PmeI and bot-HindIII-NheI, and cloned into pJET vector (ThermoFisher). pJET-Padh1 was digested with PmeI and ClaI (within pJET), and the natCM cassette of pFA6a-natCM was replaced with Padh1 using NruI and ClaI digest to generate pFA6a-Padh1. To create pFA6a-TKanAX and pFA6aTKnatAX, pFA6a-TKanMX6 and pFA6a-TKnatMX6 were 
a

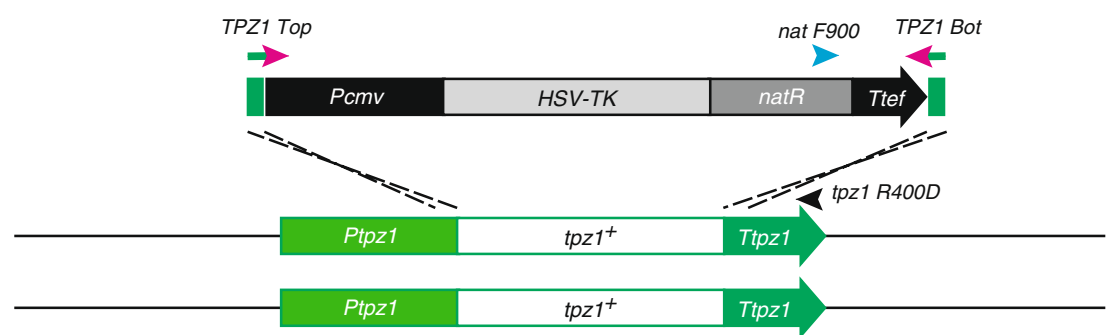

b



C



Fig. 7 Two-step mutagenesis. Schematic diagram of the two-step gene replacements and induction of point mutation of the tpzl gene. List of diagnostic primers is shown in Table 2. a PCR based tpzl gene deletion by the TKnatCX targeting fragment generated using the tpzl Top and Bot 100 base primer set (Table 1). 80-100 base of homologous sequences in the 100 base primer set targets $5^{\prime}$ and $3^{\prime}$ UTR regions of tpz $1^{+}$of one of endogenous $t p z 1^{+}$alleles. Replacement of $t p z 1^{+}$by TKnatCX was confirmed by amplification of DNA fragment using diagnostic PCR primers, nat F900 and tpz1 R400D. b Replacement of the TKnat cassette by the tpzl mutant gene. The DNA fragment containing the promoter region and the gene of $t p z 1^{+}$was amplified using primers tpzl F800U and R1745 and cloned between EcoO109l and Nhel sites of pNX3a-HA3. The tpzl gene was mutated to generate K75A mutation using the sight-directed mutagenesis method. Pvull and Pmel digested tpzl(K75A)-3XHA:kanMX6 fragment from pTpz1a4-K75A-HA3 can only recombine with TKnatCX deleted tpzl allele, as right arm homology Ttef sequence is only present in the TKnatCX cassette. $\mathbf{c}$ Integration of tpz1-K75A mutation and 3XHA tagging. Correct replacement was confirmed by amplification of DNA fragments using diagnostic PCR primer sets, kan F800 and tpz1 R400D, and tpz1 F842U and Ptef R81 (or kan R276)

digested with SpeI, and the TKan and TKnat genes were inserted at SpeI site of pFA6a-Padh1 in forward direction. To create pFA6a-HyTKAX, the HyTK-Ttef fragment was amplified from pFA6a-HyTKMX6 using primers, Hyg topHindIII and $100 \mathrm{bp}$-bot, and cloned into pFA6a between HindIII and PmeI sites, and Padh1 from pFA6a-Padh1 was inserted at HindIII site in forward direction.

The Cre expression plasmid was created as follows. The $C M V$ promoter was isolated from pAUR224 (TAKARA/ Clontech) using Tsp45I (blunt ended) and HindIII, and was inserted at NdeI (blunt ended) and HindIII site of pFA6a-13xmyc-hygMX6. The three tandem HA epitope encoding DNA fragments $(3 x H A)$ were synthesized by annealing of oligos, 3xHA top and bot, followed by DNA polymerase reaction. The 3xHA DNA fragment was generated by digest with PacI and AscI and replaced 13xmyc gene. The Cre recombinase encoding gene was cloned using primer $n l s$-Cre top-XbaI that includes $s v 40$ nuclear localization signal (NLS) at 5' linker and Cre bot-XbaI, and was digested and inserted at XbaI site downstream of $3 \mathrm{xHA}$. The resulting plasmid carried the cassette expressing 3xHA-nls-Cre from the $C M V$ promoter and was named pNXVa-HA3nlsCre. The DNA fragment containing the $S$. pombe replication origin, ars1, was isolated by AvrII digest of the $S$. pombe plasmid pKAN1. pFA6akanMX6 was digested with EcoRI and SapI, and the ars1 containing AvrII fragment was inserted following blunt ending of the cleaved sites. The resulting plasmid that carried kanMX6 and ARS1 was named pNXRa. The Cre expression cassette was isolated by EcoO109I and BglII digest and inserted into pNXRa to generate pNXRVaHA3nlsCre. 
Table 6 Oligos used for cloning and plasmid construction

\begin{tabular}{|c|c|}
\hline Name & Sequence \\
\hline Pcmv top-Pmel & atcatgaccggtttaaacCGTTACATAACTTACGGTAA \\
\hline Pcmv top-Bglll & $\begin{array}{l}\text { atcaccggtgctagcagatctcgcgaCGTTACATAAC } \\
\text { TTACGGTAA }\end{array}$ \\
\hline Tsv40 bot-Bglll & atccctaggctgcagatctGCAGTGAAAAAAATGCTTTA \\
\hline Tsv40 bot-Pmel & $\begin{array}{l}\text { agctgtacagatatcgagctcgtttaaacGCAGTGAAA } \\
\text { AAAATGCTITA }\end{array}$ \\
\hline Hyg top-HindIII & $\begin{array}{l}\text { atcctgaagcttacatgtccATGGGTAAAAAGCCTGA } \\
\text { ACTCACCGCGACG }\end{array}$ \\
\hline Hyg bot-Nhel-Clal & atcgattagctagcTACTTCTACACAGCCATCGGTCC \\
\hline Tscleu2 top-Clal-Pstl & $\begin{array}{l}\text { atcgattagctgcagtAATCCTTGCTTAAAAAGATT } \\
\text { CTCT }\end{array}$ \\
\hline Tscleu2 bot-Pmel & $\begin{array}{l}\text { agctgtacagatatcgagctcgtttaAACTCCATCAAAT } \\
\text { GGTCAGG }\end{array}$ \\
\hline $\begin{array}{l}\text { HSVtk top-Spel-Ncol- } \\
\text { HIndIII }\end{array}$ & $\begin{array}{l}\text { atcaagcttccatggaaactagtGCGTTCGACCAGGC } \\
\text { TGCGCGTT }\end{array}$ \\
\hline HSVtk bot-Xmal-Ncol & atcgattaacccatggecCGGGCAAACGTGCGCG \\
\hline Padh1 top-Pmel & $\begin{array}{l}\text { tcaggcccttgaattcgtttaaacCCTACAACAACTAA } \\
\text { GAAAATGGCTATCATGCGGAAG }\end{array}$ \\
\hline Padh1 bot-HindIII-Nhel & $\begin{array}{l}\text { atcgctagcAAgcTTCTCTTGCTTAAAGAAAAGAA } \\
\text { AAGCGAAGGCACCTGTCCACCACCC }\end{array}$ \\
\hline 100 bp-bot & GAATTCGAGCTCGTTTAAAC \\
\hline Tscadh1 For-Ascl & tgaggCGCGCCACTTCTAAATAAGCG \\
\hline Tscadh1 R110-Bglll & aagatctCCTAGCGGATCTGCCGGTAG \\
\hline Amp rev & CGACACGGAAATGTTGAATACTC \\
\hline ori-AfIIII & CCTITTGTGGCCTITGCTCACAT \\
\hline lox71-Padh1 top-Bglll & $\begin{array}{l}\text { acggttaattaagatctaccgttcgtatagcatacattatacga } \\
\text { agttatactagtcatatgCCTACAACAACTAAGAAAA } \\
\text { TGGCTATCA }\end{array}$ \\
\hline lox66-Ttef bot-Pmel & $\begin{array}{l}\text { atcgaattcgagctcgtttaaactaccgttcgtataatgtatg } \\
\text { ctatacgaagttataggcctaGGATGGCGGCGTTAG } \\
\text { TATCG }\end{array}$ \\
\hline nls-Cre top-Xbal & $\begin{array}{l}\text { atttaattaactctagaTGTACTCCACCAAAGAAGA } \\
\text { AGAGAAAGGTGCCcctagcATGGCCAATTTA } \\
\text { CTGACCGTACACCA }\end{array}$ \\
\hline Cre bot-Xbal & $\begin{array}{l}\text { aggctagcggcgcgecTCAtctagaATCGCCATCTTC } \\
\text { CAGCAGGC }\end{array}$ \\
\hline ura4 F530U-Bglll & $\begin{array}{l}\text { ggcatagatctgagctcgatatcAAGCTTAGCTACAAA } \\
\text { TCCCACTGGCTATATGT }\end{array}$ \\
\hline ura4 R190D-Pmel & $\begin{array}{l}\text { ttacctgagctcgtttaaacAAGCTTGTGATATTGACG } \\
\text { AAACTIIITGACATCTAAT }\end{array}$ \\
\hline leut F130U-BgIII & aagcttagatctCCATACGATATCCCAATCTGTAG \\
\hline leu1 R200D-Pmel & $\begin{array}{l}\text { atcgagctcgtttaaacGGATGTCGTAAATCAATTC } \\
\text { CATGC }\end{array}$ \\
\hline $\arg 3$ F130U-Bglll & aagatcTCGGCTATATGCAATCTCAC \\
\hline $\arg 3$ R130D-Pmel & aagtttaaacAGCCTGTGTCCTGCGCATA \\
\hline ade6 F300U-Bglll & aagatcTAAGGTATAACGACAACAAACGTTGC \\
\hline ade6 R150D-Pmel & $\begin{array}{l}\text { aagtttaaaCTGCTTCACAGCACATTATTCAGGAT } \\
\text { TCTT }\end{array}$ \\
\hline aur1 F333U-Bglll & $\begin{array}{l}\text { aagtcagcggcgcgccaagatctGTCAGGAAAGCTT } \\
\text { TITGCCTCT }\end{array}$ \\
\hline aur1 R280D-Pmel & $\begin{array}{l}\text { agaattcgagctcgtttaaaCAAATACCTATACATCAC } \\
\text { ACTGGAA }\end{array}$ \\
\hline
\end{tabular}

Table 6 Oligos used for cloning and plasmid construction (Continued)

\begin{tabular}{|c|c|}
\hline $3 \times H A$ top & $\begin{array}{l}\text { atccccgggttaattaacgctagcatgTACCCATACGAT } \\
\text { GTTCCTGACTATGCGGGCTACCCGTATGACGTG } \\
\text { CCGGACTATGCAGGAGCCTATCCTATGACGTT } \\
\text { CCAGA }\end{array}$ \\
\hline $3 \times H A$ bot & $\begin{array}{l}\text { aggctagcggcgcgccTCAtctagaACCAGCGTAATC } \\
\text { TGGAACGTCATAAGGATAGGCTCCTGCATAGTC } \\
\text { CGGCACGTCATACGGGTAGCCCGCATAGTCAGG } \\
\text { AACATCG }\end{array}$ \\
\hline 3XPK-H6 top & $\begin{array}{l}\text { ggatccatatgTTAATTAACGCTAGCGGTAAGCCTA } \\
\text { TTCCTAACCCTCTIITGGGTCTCGATTCTACAGG } \\
\text { TGGAAAACCAATCCCCAACCCACTCCTCGGCCT } \\
\text { TGACTCAACTGGCAAAC }\end{array}$ \\
\hline $3 \times P K-H 6$ bot & $\begin{array}{l}\text { agatctggcgcgcctaggatggtgatggtgatgatgtctaga } \\
\text { GGTACTATCTAATCCAAGTAAAGGATITGTATG } \\
\text { GGTITGCCAGTTAGTCAAGGCCGAGGAGTGGG } \\
\text { TTGGGGATTGG }\end{array}$ \\
\hline 3xFlag top & $\begin{array}{l}\text { taacgctagcatgGGTGACTACAAGGACGACGATG } \\
\text { ACAAGGGAGATTACAAAGATGACGACGATAA } \\
\text { GGACTACAAGGACGACGATGACAAGtctagaTG } \\
\text { Agg }\end{array}$ \\
\hline 3xFlag bot & $\begin{array}{l}\text { cgcgecTCAtctagaCTTGTCATCGTCGTCCTTGTAG } \\
\text { TCCTAATCGTCGTCATCIITGTAATCTCCCTTGTC } \\
\text { ATCGTCGTCCTTGTAGTCACCcatgctagcgttaat }\end{array}$ \\
\hline tpz1 F800U-Pvull-EcoO & atcaggecctcagCTGTTCAGCACGGTACCAAG \\
\hline tpzl R1745-Nhel & gttgcagctagcGCTTTTGTTCGAAACTCCTCTAT \\
\hline
\end{tabular}

Auxotrophic markers $\mathrm{ura}^{+}, \mathrm{leu1^{+ }}, \mathrm{arg}^{+}{ }^{+}$and $\mathrm{ade6}^{+}$ were cloned from $S$. pombe genomic DNA using corresponding primer sets listed in Table 6 , and cloned into pJET vector. To generate Cre-expression vectors with various selection cassettes, auxotrophic markers leu1 ${ }^{+}$, $\arg ^{+}$, $\mathrm{ura}^{+}$and $\mathrm{ade}^{+}$and drug selection markers $T \mathrm{ka}-$ nAX, hygMX6, HyTkAX, natMX6, TKnatAX and aur ${ }^{R}$ from the plasmid pAUR224 were amplified with primers containing with $B g l \mathrm{II}$ and PmeI sites and cloned into pJET vector (ThermoFisher). The cloned cassettes were digested with BglII and PmeI and replaced with kanMX6 of pNXRVa-HA3nls-Cre. For the systematic reason, the drug resistant cassettes for G418, hygromycin B, clonNat, and aureobasidin A and auxotrophic markers for uracil, arginine, leucine, and adenine were assigned as ' $a$ ', 'b', 'c', 'j,' 'd, 'g,' 'h' and 'k', respectively. Through the cloning process, we found point mutation (G201T) in the Zeocin resistant gene that substitutes Tryptophan at the position 67 to Cysteine (W67C), but the cassette retained resistance to Zeocin (Fig. 1b).

The series of the C-terminal tagging plasmids, pNX3 was constructed as follows. The terminator of the $S$. cerevisiae adh1 was cloned from the genomic DNA using primers, Tscadh1 For-AscI and Tscadh1 R110$B g l I I$, and inserted into pNXRa after digestion with $A s c \mathrm{I}$ and BglII. ARS1 sequence was removed by digest of pNXRa with EcoRI and AvrII and ligation following 
blunt ending of the cleaved sites. Unlike pFA6a C-tag plasmids, this resulting plasmid, named pNX3a, lacks $I$ SceI after Tadh1 and ClaI, EcoRV, SpeI, SfiI, NotI, SacII, HpaI and SapI after kanMX6. To generate pNX3a$\mathrm{HA} 3$, the $3 \times H A$ fragment was produced by digest as mentioned above and inserted into pNX3a following $P a c \mathrm{I}$ and $A s c \mathrm{I}$ digest. Likewise, the three tandem $P K$ fragment (3xPK) was generated by annealing of oligos, $3 \times P K-H 6$ top and bot, followed by DNA polymerase reaction. To generate pNX3a-PK3, the 3xPK fragment was obtained by digest and replaced 3xHA of pNX3aHA3 after PacI-XbaI digest. Three tandem FLAG fragment (3xFL) was generated by annealing of oligos, $3 x$ Flag top and bot, followed by 5 ' end phosphorylation. To generate pNX3a-FL3, the 3xFL fragments were inserted into pNX3a after $P a c I$ and AscI digest. The kanMX6 cassette was replaced by other selection markers using BglII and PmeI digest. All generated plasmids for the $\mathrm{C}$-terminus tagging are listed in Table 5.

\section{PCR amplification for gene targeting fragments}

For amplification of the deletion fragments using pFA6a series, the primer set (Top and Bot) was used. Top primer is composed of $80-100$ base forward sequence upstream of the start codon of the target gene at the five prime end and the vector annealing sequence CGGATCCCCGGGTTAATTAA at the three prime end. Bot primer is composed of the complement sequence of downstream 80 100 base sequence after the stop codon of the target gene at the five prime end and vector annealing sequence GAATTCGAGCTCGTTTAAAC at the three prime end.

For $\mathrm{C}$-terminus tagging using $\mathrm{pNX} 3$ series, the $\mathrm{C}$-tag specific Tag primer and the deletion Bot primer were used. The annealing sequence of Tag primer is the same as Top primer for deletion but targeting sequence is 80 100 base upstream from the end of the gene excluding the stop codon. These oligos were synthesized by Integrated DNA Technologies Inc. (IDT) using standard desalting purification service. Details of primer design have been described previously [2].

Three hundred microliter of PCR mixtures contained $30 \mu \mathrm{l}$ of 10xbuffer I, $6 \mu \mathrm{l}$ of $10 \mathrm{mM}$ dNTPs and $1.5 \mu \mathrm{l}$ of long template polymerase mixture (Roche) with $0.75 \mu \mathrm{l}$ of pFA6a or pNX3 template vector (approximately 50-500 ng/ $\mu \mathrm{l}$, obtained using standard miniprep kit), and $1.5 \mu \mathrm{l}$ each of $100 \mu \mathrm{M}$ primers. Premix was aliquoted into $50 \mu \mathrm{l}$ and subjected to PCR $\left[94{ }^{\circ} \mathrm{C} 30 \mathrm{sec}\right.$, $50{ }^{\circ} \mathrm{C} 2 \mathrm{~min}, 68^{\circ} \mathrm{C} 5 \mathrm{~min}$ for 5 cycles, followed by $94{ }^{\circ} \mathrm{C}$ $30 \mathrm{sec}, 55^{\circ} \mathrm{C} 1 \mathrm{~min}, 68^{\circ} \mathrm{C} 5 \mathrm{~min}$ for 30 cycles, finishing with $\left.68{ }^{\circ} \mathrm{C} 10 \mathrm{~min}\right]$. PCR products were combined, ethanol precipitated, and reconstituted in $10 \mu \mathrm{l}$ of TE (10 mM Tris-HCl 1 mM EDTA pH8.0).

\section{PCR-based gene targeting}

Fission yeast transformation was performed as described [2] with minor changes. Logarithmically growing cells $\sim 10^{7}$ cells $/ \mathrm{ml} \quad\left(\mathrm{O}_{600}=0.5-0.8\right)$ were harvested, and washed once with LiOAc solution (100 mM Lithium Acetate pH7.5 in TE). Cell pellets were suspended in equal volume of LiOAc solution to make competent cells. $50 \mu \mathrm{l}$ of the competent cells were mixed with the gene targeting PCR product, $130 \mu \mathrm{l}$ of $40 \%$ PEG solution ( $40 \%$ of PEG4000 in LiOAc solution) was added to the cells and mixed gently by tapping. Cells were incubated at $32{ }^{\circ} \mathrm{C}$ for 1-4 h. After adding $21.5 \mu \mathrm{l}$ of DMSO, and the cells were heat-shocked at $42{ }^{\circ} \mathrm{C}$ for $5 \mathrm{~min}$, precipitated briefly and washed once with YES media. The transformed cells were directly plated on EMM media for auxotrophic selection or YES/EMM media for Aureobasidin B (TAKARA/Clontech) selection. For other drug selection, transformed cells were recovered in YES media for $4 \mathrm{~h}$ in shaking incubator or overnight on the plate, and transferred to YES plate containing G418 (FORMEDIUM), Zeocin (Invivogen), Hygromycine B (FORMEDIUM and Roche) or ClonNat/ Nourseothricin (WERNER BioAgents). YES can be replaced with PMG media.

\section{Cre-loxP counter selection}

To remove loxP-flanked $T K$ fusion cassette, cells were transformed with any Cre expression vector (see transformation procedure in previous section). After heat- shock process, cells were directly plated on FdU containing YES plate. FdU-resistant colonies were re-streaked on a fresh FdU plate to isolate a single colonies. Loss of $T K$ fusion cassette and the Cre expression vector were confirmed by sensitivity to the drugs. Alternatively, pNXRVj-HACre was used for Cre expression, the transformants carrying Cre vector were selected by resistance to aureobasidin $\mathrm{B}$. The resistant colonies were re-streaked on YES plates, and colonies that lost the drug resistance were isolated.

\section{Southern blot}

Telomere Southern blotting was performed as described previously [28]. Genomic DNA was prepared 2 weeks after generation of the strains unless indicated. Equal amounts of EcoRI digested DNA fragments were separated on a $1 \%$ agarose gel and subjected to Southern blotting with a telomere probe.

\section{Funding}

This work is supported mainly by the European Research Council (281722HRMCB) and Cancer Research UK (C36439/A12097), and partly by the Cancer Research UK - UCL Centre. These funding bodies had no role in design, collection, and analysis of the study and preparation of the manuscript.

Availability of data and materials

All generated plasmids and their full sequence and map are available from Addgene (www.addgene.com). 


\section{Authors' contributions}

$\mathrm{KT}$, HA and VM were responsible for study design, experimental work, data analysis. KT and SRP constructed plasmids and verified by sequencing. HA and VM performed stress and drug resistance assay. VM, HA, LCC, SRP, CAA and $K T$ performed strain construction using generated plasmids and confirmed their functions. LCC performed Tpz1 PK tagging and strain characterization. SRP and VM performed Cre/loxP experiment. LCC, CAA and SRP conducted the two-step point mutation of the tpzl gene. KT drafted and all authors were responsible for manuscript preparation. All authors read and approved the final manuscript.

\section{Competing interests}

The authors declare that they have no competing interests.

\section{Consent for publication}

Not applicable.

\section{Ethics approval and consent to participate}

Not applicable.

Received: 12 April 2016 Accepted: 21 October 2016

Published online: 08 November 2016

\section{References}

1. Hoffman CS, Wood V, Fantes PA. An ancient yeast for young geneticists: a primer on the schizosaccharomyces pombe model system. Genet. 2015;201(2):403-23.

2. Bahler J, Wu JQ, Longtine MS, Shah NG, McKenzie 3rd A, Steever AB, Wach A, Philippsen P, Pringle JR. Heterologous modules for efficient and versatile PCR-based gene targeting in Schizosaccharomyces pombe. Yeast. 1998;14(10):943-51.

3. Siam R, Dolan WP, Forsburg SL. Choosing and using Schizosaccharomyces pombe plasmids. Methods. 2004;33(3):189-98.

4. Hentges P, Van Driessche B, Tafforeau L, Vandenhaute J, Carr AM. Three novel antibiotic marker cassettes for gene disruption and marker switching in Schizosaccharomyces pombe. Yeast. 2005;22(13):1013-9.

5. Sato M, Dhut S, Toda T. New drug-resistant cassettes for gene disruption and epitope tagging in Schizosaccharomyces pombe. Yeast. 2005;22(7):583-91.

6. Beach D, Nurse P. High-frequency transformation of the fission yeast Schizosaccharomyces pombe. Nature. 1981;290(5802):140-2.

7. Grimm C, Kohli J, Murray J, Maundrell K. Genetic engineering of Schizosaccharomyces pombe: a system for gene disruption and replacement using the ura4 gene as a selectable marker. Mol Gen Genet MGG. 1988:215(1):81-6.

8. Burke JD, Gould KL. Molecular cloning and characterization of the Schizosaccharomyces pombe his3 gene for use as a selectable marker. Mol Gen Genet MGG. 1994;242(2):169-76.

9. Waddell $S$, Jenkins JR. arg3+, a new selection marker system for Schizosaccharomyces pombe: application of ura4+ as a removable integration marker. Nucleic Acids Res. 1995;23(10):1836-7.

10. Jacobs JZ, Ciccaglione KM, Tournier V, Zaratiegui M. Implementation of the CRISPR-Cas9 system in fission yeast. Nat Commun. 2014;5:5344.

11. Paquet D, Kwart D, Chen A, Sproul A, Jacob S, Teo S, Olsen KM, Gregg A Noggle S, Tessier-Lavigne M. Efficient introduction of specific homozygous and heterozygous mutations using CRISPR/Cas9. Nature. 2016:533(7601):125-9.

12. Amelina H, Subramaniam S, Moiseeva V, Armstrong CA, Pearson SR, Tomita K. Telomere protein Rap1 is a charge resistant scaffolding protein in chromosomal bouquet formation. BMC Biol. 2015;13:37.

13. Saka Y, Esashi F, Matsusaka T, Mochida S, Yanagida M. Damage and replication checkpoint control in fission yeast is ensured by interactions of Crb2, a protein with BRCT motif, with Cut5 and Chk1. Genes Dev. 1997:11(24):3387-400.

14. Catlett MG, Forsburg SL. Schizosaccharomyces pombe Rdh54 (TID1) acts with Rhp54 (RAD54) to repair meiotic double-strand breaks. Mol Biol Cell. 2003; 14(11):4707-20

15. Kiely J, Haase SB, Russell P, Leatherwood J. Functions of fission yeast orp2 in DNA replication and checkpoint control. Genet. 2000;154(2):599-607.

16. Longley DB, Harkin DP, Johnston PG. 5-fluorouracil: mechanisms of action and clinical strategies. Nat Rev Cancer. 2003:3(5):330-8.

17. Lupton SD, Brunton LL, Kalberg VA, Overell RW. Dominant positive and negative selection using a hygromycin phosphotransferase-thymidine kinase fusion gene. Mol Biol Cell. 1991;11(6):3374-8.
18. Schwartz F, Maeda N, Smithies O, Hickey R, Edelmann W, Skoultchi A, Kucherlapati R. A dominant positive and negative selectable gene for use in mammalian cells. Proc Natl Acad Sci U S A. 1991;88(23):10416-20.

19. Hodson JA, Bailis JM, Forsburg SL. Efficient labeling of fission yeast Schizosaccharomyces pombe with thymidine and BUdR. Nucleic Acids Res. 2003;31(21):e134.

20. Martinho RG, Lindsay HD, Flaggs G, DeMaggio AJ, Hoekstra MF, Carr AM, Bentley NJ. Analysis of Rad3 and Chk1 protein kinases defines different checkpoint responses. EMBO J. 1998;17(24):7239-49.

21. Hua $H$, Kearsey SE. Monitoring DNA replication in fission yeast by incorporation of 5-ethynyl-2'-deoxyuridine. Nucleic Acids Res. 2011;39(9):e60

22. Akamatsu $Y$, Dziadkowiec D, Ikeguchi M, Shinagawa H, Iwasaki H. Two different Swi5-containing protein complexes are involved in mating-type switching and recombination repair in fission yeast. Proc Natl Acad Sci U S A. 2003;100(26):15770-5.

23. McNabb DS, Pak SM, Guarente L. Cassette for the generation of sequential gene disruptions in the yeast Schizosaccharomyces pombe. BioTechniques. 1997;22(6):1134-9.

24. Iwaki T, Takegawa K. A set of loxP marker cassettes for Cre-mediated multiple gene disruption in Schizosaccharomyces pombe. Biosci Biotechnol Biochem. 2004;68(3):545-50.

25. Toh-e A. Construction of a marker gene cassette which is repeatedly usable for gene disruption in yeast. Curr Genet. 1995;27(4):293-7.

26. Zhang Z, Lutz B. Cre recombinase-mediated inversion using lox66 and lox71: method to introduce conditional point mutations into the CREBbinding protein. Nucleic Acids Res. 2002;30(17):e90.

27. Hashida-Okado T, Yasumoto R, Endo M, Takesako K, Kato I. Isolation and characterization of the aureobasidin A-resistant gene, aur1R, on Schizosaccharomyces pombe: roles of Aur1p + in cell morphogenesis. Curr Genet. 1998;33(1):38-45.

28. Armstrong CA, Pearson SR, Amelina H, Moiseeva V, Tomita K. Telomerase activation after recruitment in fission yeast. Current Biol. 2014;24(17):2006-11.

29. Jun HI, Liu J, Jeong H, Kim JK, Qiao F. Tpz1 controls a telomerasenonextendible telomeric state and coordinates switching to an extendible state via Ccq1. Genes Dev. 2013:27(17):1917-31.

30. Harland JL, Chang YT, Moser BA, Nakamura TM. Tpz1-Ccq1 and Tpz1-Poz1 interactions within fission yeast shelterin modulate Ccq1 Thr93 phosphorylation and telomerase recruitment. PLoS Genet. 2014;10(10):e1004708.

31. Miyoshi T, Kanoh J, Saito M, Ishikawa F. Fission yeast Pot1-Tpp1 protects telomeres and regulates telomere length. Science. 2008;320(5881):1341-4.

32. Miyagawa K, Low RS, Santosa V, Tsuji H, Moser BA, Fujisawa S, Harland JL, Raguimova ON, Go A, Ueno M, et al. SUMOylation regulates telomere length by targeting the shelterin subunit Tpz1(Tpp1) to modulate shelterin-Stn interaction in fission yeast. Proc Natl Acad Sci U S A. 2014;111(16):5950-5.

\section{Submit your next manuscript to BioMed Central and we will help you at every step:}

- We accept pre-submission inquiries

- Our selector tool helps you to find the most relevant journal

- We provide round the clock customer support

- Convenient online submission

- Thorough peer review

- Inclusion in PubMed and all major indexing services

- Maximum visibility for your research

Submit your manuscript at www.biomedcentral.com/submit
Biomed Central 